\title{
Triggering on hadronic signatures in the ATLAS experiment - Developments for 2017 and 2018
}

\author{
Emanuel Gouveia ${ }^{1 *}$, on behalf of the ATLAS Collaboration** \\ ${ }^{1}$ LIP, Departamento de Física, Universidade do Minho, 4710-057 Braga, Portugal
}

\begin{abstract}
Hadronic signatures are critical to the ATLAS physics program, and are used extensively for both the Standard Model measurements and searches for new physics. These signatures include generic quark and gluon jets, as well as jets originating from $b$-quarks or the decay of massive particles (such as electroweak bosons or top quarks). Additionally, missing transverse momentum from non-interacting particles provides an interesting probe in the search for new physics beyond the Standard Model. Developing trigger selections that target these events is a huge challenge at the LHC due to the enormous rates associated with hadronic signatures. This challenge is exacerbated by the amount of pile-up activity, which continues to grow. In order to address these challenges, several new techniques were developed to significantly improve the potential of the 2017 dataset. An overview of how we trigger on hadronic signatures at the ATLAS experiment is presented, outlining the challenges of hadronic object triggering and describing the improvements performed over the course of the Run 2 LHC data-taking program. The performance in Run 2 data is shown, including demonstrations of the new techniques being used in 2017. We also discuss further critical developments implemented for the rest of Run 2 and their performance in early 2018 data.
\end{abstract}

\section{Introduction}

The ATLAS experiment [1] is a multi-purpose detector at the LHC. Its design was driven mainly by the potential to search for the Standard Model (SM) Higgs boson, before its mass was known, and to search for new particles Beyond the Standard Model (BSM), such as heavier gauge or Higgs bosons, dark matter candidates and supersymmetric particles. All the production processes of such particles may result in final states with jets, coming from the hadronisation of strongly interacting particles, or missing transverse energy, due to neutrinos or new particles with very weak interactions.

The ATLAS trigger system must reduce the proton-proton bunch crossing rate of the LHC down to an average of $1 \mathrm{kHz}$. In hadron colliders such as the LHC, every hard-scatter process can occur with the emission of additional jets with considerable transverse momentum $\left(p_{\mathrm{T}}\right)$. Moreover, since protons travel in compact bunches, there are usually many inelastic interactions per bunch crossing (pile-up), the majority of which correspond to QCD-mediated di-jet production. The average pile-up in ATLAS was 37.8 (37.3) interactions per bunch-crossing

\footnotetext{
*Speaker. e-mail: e.gouveia@cern.ch

${ }^{* *}$ From ATL-DAQ-PROC-2018-040. Published with permission by CERN.
} 
in 2017 (2018, through 10 September 2018) [2]. This makes triggering on every event with jets not feasible. The same is true for the missing transverse energy $\left(E_{\mathrm{T}}^{\mathrm{miss}}\right)$ computed from the hadronic component of the event. This is because the $p_{\mathrm{T}}$ balance condition is not met exactly, due to limited resolution in the hadronic calorimeters. If the vector $p_{\mathrm{T}}$ imbalances from several pile-up interactions happen to sum constructively or the $p_{\mathrm{T}}$ of a jet is incorrectly measured, this may result in considerable reconstructed $E_{\mathrm{T}}^{\mathrm{miss}}$ in the event.

The main challenge for the jet and $E_{\mathrm{T}}^{\text {miss }}$ trigger signatures is then to reconstruct features as closely to the offline reconstruction as possible, using only the limited information available in the trigger. This should be achieved while ensuring rates do not increase faster than linearly as a function of pile-up.

\section{Jet trigger}

\subsection{Level-1 jet trigger}

The first rate-reducing step of the ATLAS trigger system is the Level-1 trigger (L1). The L1 jet trigger [3] is based on coarse granularity information from calorimeters. Signals from the calorimeters are aggregated according to detector segments with approximate dimension $0.2 \times$ 0.2 in the $\eta-\phi$ plane, called jet elements. The dimensions of the jet elements are more irregular in the forward $(|\eta|>2.5)$ region. Jet elements are built separately for the electromagnetic and hadronic calorimeters. Regions of interest (RoIs) are defined as windows of $4 \times 4$ jet elements (i.e., $0.8 \times 0.8$ in $\eta-\phi$ ) if they meet two conditions: the summed $E_{\mathrm{T}}$ from the hadronic calorimeter over their core $(2 \times 2$ central jet elements $)$ is at a local maximum and the summed $E_{\mathrm{T}}$ over the whole RoI is above a certain predefined threshold (see Figure 1).

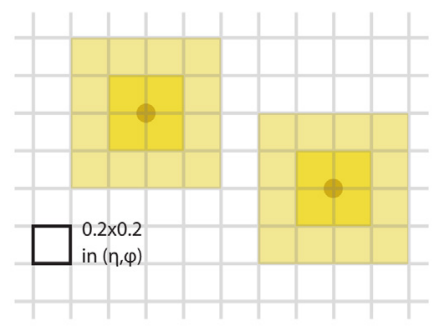

Figure 1. Schematic representation of L1 jet elements (grid), RoIs ( $4 \times 4$ yellow squares) and cores $\left(2 \times 2\right.$ dark yellow squares). The summed $E_{\mathrm{T}}$ from the hadronic calorimeter within the cores must be a local maximum for the RoI to be built. The RoI coordinates are those of the centres of the small circles.

\subsection{High-level jet trigger}

\subsubsection{Jet reconstruction}

The high-level trigger (HLT) is the final rate-reducing step of the ATLAS trigger system. For the jet HLT, information with full calorimeter granularity is available [4]. Taking the signals from calorimeter cells as input, a three-dimensional topological clustering algorithm is run. Jets are reconstructed with the anti- $k_{t}$ algorithm using the previously built topological clusters as input. For small- $R$ jets, a parameter $R=0.4$ is used, while large- $R$ jets have $R=1.0$. The clusters may or may not be weighted prior to jet reconstruction, with weights which depend on their longitudinal depth and energy density. The default procedure is to not apply these weights when reconstructing small- $R$ jets and to apply them when reconstructing large- $R$ jets. 


\subsubsection{Jet calibration}

A calibration sequence is applied to the reconstructed jets as an attempt to recover the expected properties of a corresponding particle-level jet. This is done in a manner as close as possible to the offline jet calibration, within the information constraints of the trigger.

The first step of the calibration of small- $R$ jets in ATLAS [5], which is the origin correction, depends on vertex reconstruction. This step is not performed in the trigger because tracks are in general not available. The first calibration step applied in the trigger is the jet area based pile-up subtraction, in which the contribution from the median $p_{\mathrm{T}}$ density in the event is removed from jets, proportionally to their area. Residual pile-up corrections depend in part on the number of primary vertices, which again requires tracks, and are thus not applied in the trigger. The following step is the jet energy scale calibration, which corrects the jet energy and $\eta$ to those expected of a corresponding particle-level jet. Then, the global sequential calibration (GSC) is applied. The offline GSC combines calorimeter, tracking and muon-segment variables to account for the different detector responses to quark- or gluoninitiated jets. In the trigger, a reduced version is used, relying on calorimeter information only by default and, in some cases, also using track information. The final step is the in situ calibration, applied only to data, in which remaining differences between data and MC are covered.

For large- $R$ jets [6], the first calibration step is jet grooming, using the trimming algorithm from [7], to reduce dependence on pile-up and the underlying event. This method removes small- $R$ subjets of the large- $R$ jet if they contribute with a fraction smaller than $f$ to the large$R$ jet $p_{\mathrm{T}}$. The parameter $f$ is set to $5 \%$ in the offline calibration and to $4 \%$ in the trigger. This looser threshold in the trigger prevents a trigger inefficiency that was observed if the same threshold was used as in the offline. Next, an MC-based correction is applied to the groomed jets. Besides correcting the energy and $\eta$ of the jets, as happened in small- $R$ jets, it also corrects their mass. Currently, the in situ step is not applied to large- $R$ jets [6].

The default trigger jet calibration sequences for small- $R$ and large- $R$ jets are summarized in Figure 2, with the main differences with respect to offline highlighted.

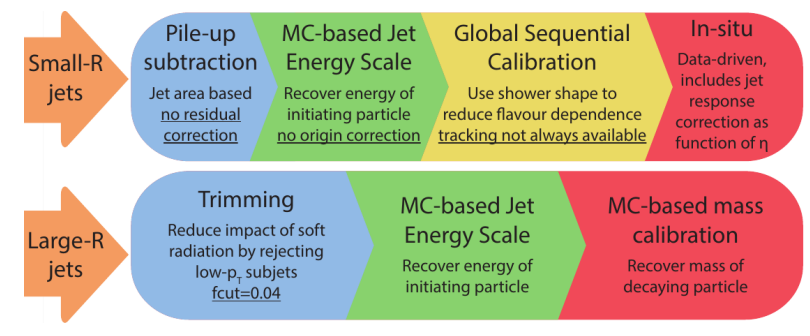

Figure 2. Default calibration steps applied to small- $R$ and large- $R$ trigger jets. The main differences with respect to offline are underlined.

\section{$3 E_{\mathrm{T}}^{\text {miss }}$ trigger}

The $\mathrm{L} 1 E_{\mathrm{T}}^{\mathrm{miss}}$ trigger [8] is based on trigger towers, which are projective calorimeter segments with approximate dimensions $0.1 \times 0.1$ in $\eta-\phi$ (larger and less regular in the forward region). The energy of the trigger towers is calibrated at the electromagnetic energy scale: it correctly reconstructs the energy deposited by particles in an electromagnetic shower but underestimates the energy deposited by hadrons. The $E_{\mathrm{T}}^{\text {miss }}$ is estimated through a vectorial sum of the transverse components of such trigger towers, over the full calorimeter acceptance. 
Several algorithms have been used for $E_{\mathrm{T}}^{\mathrm{miss}}$ computation at the HLT [4]. The recommended ones during the Run 2 of the LHC have been the jet-based algorithm ( $m h t)$ and the pile-up fit algorithm (pufit). The $m h t$ algorithm computes the $E_{\mathrm{T}}^{\text {miss }}$ as the negative of the vectorial sum of the $p_{\mathrm{T}}$ of small- $R$ jets, calibrated up to the MC-based jet energy scale step. The pufit algorithm uses 112 trigger towers of approximate dimension $0.7 \times 0.8$ as input. The $E_{\mathrm{T}}$ of each tower is obtained from a sum of $E_{\mathrm{T}}$ of topological clusters within the tower. Then, high- $E_{\mathrm{T}}$ towers are separated from low- $E_{\mathrm{T}}$ towers, with the threshold being determined on an event-by-event basis, from the mean and variance of tower $E_{\mathrm{T}}$. If there is at least one high- $E_{\mathrm{T}}$ tower, a fit is performed to estimate the pile-up contribution to the high- $E_{\mathrm{T}}$ towers. It uses the low- $E_{\mathrm{T}}$ towers as input and, taking resolution into account, imposes a pile-up energy density uniform in $\phi$ and smooth in $\eta$, with a null contribution to the total $E_{\mathrm{T}}^{\text {miss }}$. The $E_{\mathrm{T}}^{\text {miss }}$ is measured from the high- $E_{\mathrm{T}}$ towers, after subtracting the fitted pile-up contribution.

\section{Developments for 2017 and 2018}

\subsection{L1 jet cone algorithm}

The usual jet RoI definition with an $\eta-\phi$ dimension $0.8 \times 0.8$ is not adequate to trigger on the large- $R$ (for example $R=1.0$ ) jets that are relevant to physics analyses. These jets usually result from the decay of heavy particles and exhibit substructure. If the subjets within the large- $R$ jet are not close enough, the $E_{\mathrm{T}}$ within the defined RoI(s) can be significantly smaller than that of the large- $R$ jet. An additional algorithm was introduced for 2017 data taking to address this inefficiency [9]. It is implemented in the ATLAS Level-1 topological trigger processor (L1Topo) [10], a system capable of combining information from individual objects and processing it into event-level information. For each RoI, this algorithm searches for other RoIs within 1.0 in $\Delta R\left(\Delta R=\sqrt{(\Delta \eta)^{2}+(\Delta \phi)^{2}}\right)$ separation from the original one and sums the

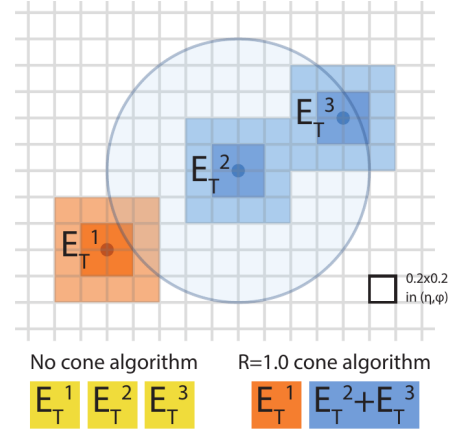

(a)

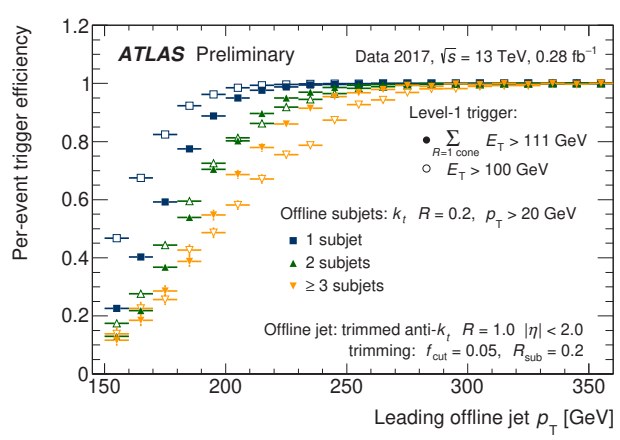

(b)

Figure 3. (a) L1 jet cone algorithm with $R=1.0$. The RoIs 2 and 3 may come from the decay of a heavy boson to quarks, for example. They would be clustered together by a large- $R$ jet algorithm into a high- $p_{\mathrm{T}}$ jet, but in L1 trigger they give rise to two lower- $E_{\mathrm{T}}$ RoIs. The cone algorithm adds their $E_{\mathrm{T}}$, resulting in a total $E_{\mathrm{T}}$ closer to the large- $R$ jet $p_{\mathrm{T}}$. (b) Efficiency of the L1 trigger, both with and without the cone algorithm, as a function of the offline leading large- $R$ jet $p_{\mathrm{T}}$ [11]. The $E_{\mathrm{T}}$ threshold with the cone algorithm is adjusted to $111 \mathrm{GeV}$ to match the same rate as the $100 \mathrm{GeV}$ threshold without the cone algorithm. 
$E_{\mathrm{T}}$ of such regions. The trigger selection is then applied based on these $E_{\mathrm{T}}$ sums and not on the RoI $E_{\mathrm{T}}$ (see Figure 3a). In Figure 3b, efficiency curves are shown for L1 jet triggers, both with and without the cone algorithm. The $E_{\mathrm{T}}$ threshold with the cone algorithm is adjusted to $111 \mathrm{GeV}$ to match the same rate as the $100 \mathrm{GeV}$ threshold without the cone algorithm. For jets with multiple subjets, a desireable sharpening of the turn-on curve is visible, while for jets with a single subjet, only a shift of the turn-on point to a higher $p_{\mathrm{T}}$ value is visible.

\subsection{Improved jet calibration}

In HLT small- $R$ jet calibration, the GSC and in situ steps were added for 2017 data taking [9]. This brings the trigger jets much closer to offline jets, which is evident in Figure 4a. Efficiencies of an unprescaled HLT small- $R$ single jet trigger are compared, for the 2016 calibration, the 2017 calibration with calorimeter-only GSC (default), and the 2017 calibration with the track-dependent GSC (only available for some thresholds and $b$-jet triggers). The

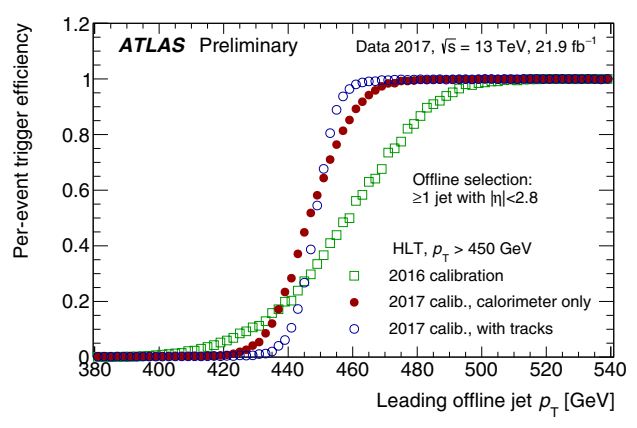

(a)

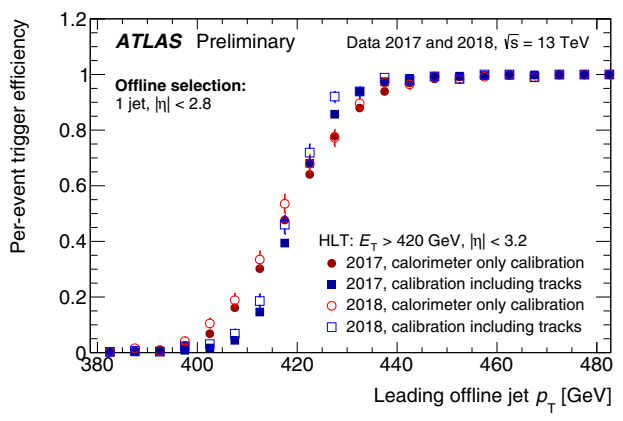

(b)

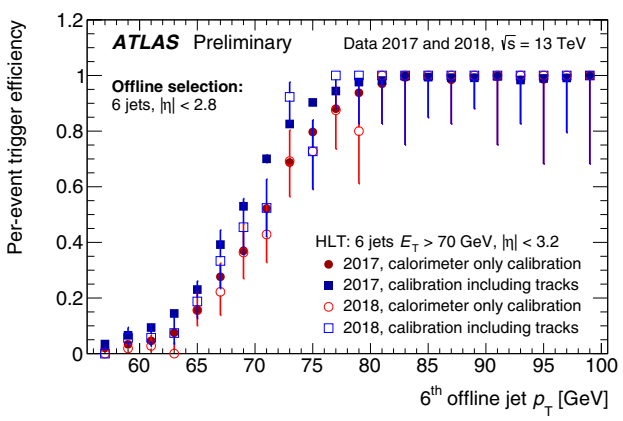

(c)

Figure 4. (a) Efficiencies of an unprescaled small- $R$ single jet trigger as a function of the leading offline jet $p_{\mathrm{T}}$, for the 2016 calibration, the 2017 calibration without tracks (default), and the 2017 calibration with tracks [11]. (b) Efficiencies of an unprescaled small- $R$ singlejet trigger as a function of the offline leading jet $p_{\mathrm{T}}$, comparing 2017 and 2018 data, with or without track-based corrections included in GSC [11]. (c) Efficiencies of an unprescaled small- $R$ six-jet trigger as a function of the offline 6th leading jet $p_{\mathrm{T}}$, comparing 2017 and 2018 data, with or without track-based corrections included in GSC [11]. 
full efficiency point is reached for significantly lower offline $p_{\mathrm{T}}$ in 2017 with respect to 2016. Figures $4 \mathrm{~b}$ and $4 \mathrm{c}$ show efficiency curves comparing the calorimeter-only GSC to the full GSC in 2017 and 2018 data separately: in Figure 4b for a single jet trigger and in Figure $4 \mathrm{c}$ for a six-jet trigger. The curves are similar between the two years, and there is a visibly sharper turn-on when track-based information is included in the GSC.

\subsection{Mass cut in large- $R$ trimmed jets}

For large- $R$ jets, the trimming procedure was also introduced for 2017 data taking, replacing the previously used area based pile-up subtraction. Large- $R$ jets are usually regarded in analyses as candidates for boosted hadronically decaying heavy $\left(m \gtrsim m_{W}\right)$ particles. Applying a mass cut to large- $R$ jets at the trigger level rejects most of the multijet background, while keeping the signal-like jets. Triggers with such a cut were added as a complement to the ATLAS jet trigger menu for 2017 data taking [9]. In Figure 5a, the efficiency of large- $R$ trimmed jet triggers is shown, with and without a mass cut at $35 \mathrm{GeV}$. The $35 \mathrm{GeV}$ mass cut allows for a $40 \mathrm{GeV}$ decrease of the $E_{\mathrm{T}}$ threshold while meeting the trigger menu rate limitations. Figure $5 \mathrm{~b}$ shows the efficiency of a large- $R$ dijet trigger with a $30 \mathrm{GeV}$ mass cut, as a function of the mass of the second leading offline large- $R$ jet. Full efficiency is reached for masses close to $50 \mathrm{GeV}$.

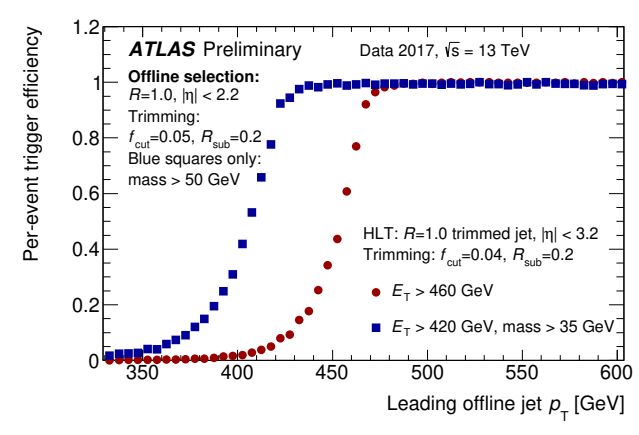

(a)

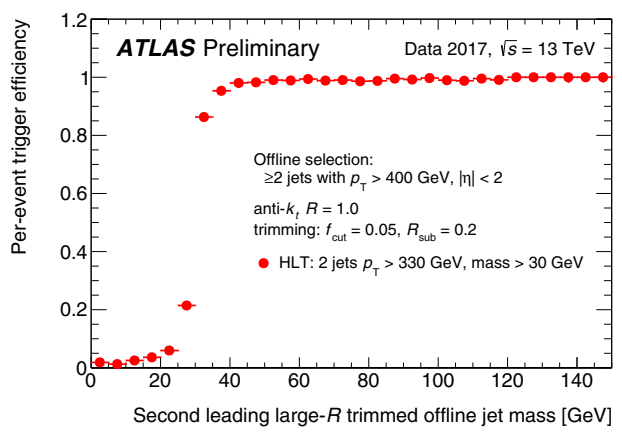

(b)

Figure 5. (a) Efficiency of large- $R$ trimmed jet triggers, with and without a mass cut at 35 $\mathrm{GeV}$, as a function of the offline leading large- $R$ trimmed jet $p_{\mathrm{T}}[11]$. (b) Efficiency of a large- $R$ dijet trigger with a $30 \mathrm{GeV}$ mass cut as a function of the mass of the second leading offline large- $R$ jet [11].

\section{4 pufit algorithm in $E_{\mathrm{T}}^{\mathrm{miss}}$}

The use of the pufit algorithm in the $E_{\mathrm{T}}^{\text {miss }}$ trigger became the primary recommended option in 2017 [9]. In Figure 6a, the efficiency of L1, mht and pufit $E_{\mathrm{T}}^{\text {miss }}$ triggers is compared. Events shown passed a $W \rightarrow \mu \nu$ selection. Muons are treated as invisible objects for the represented offline $E_{\mathrm{T}}^{\text {miss }}$, since the same is true for the triggers concerned. The superior performance of pufit is visible by the steeper turn-on. In Figure 6b, the total trigger cross-section is shown for an $m h t E_{\mathrm{T}}^{\text {miss }}$ trigger and a pufit trigger, as a function of the mean number of interactions per bunch-crossing $\langle\mu\rangle$. The rate increase is slower for pufit, which makes it more stable with respect to increasing luminosity. 


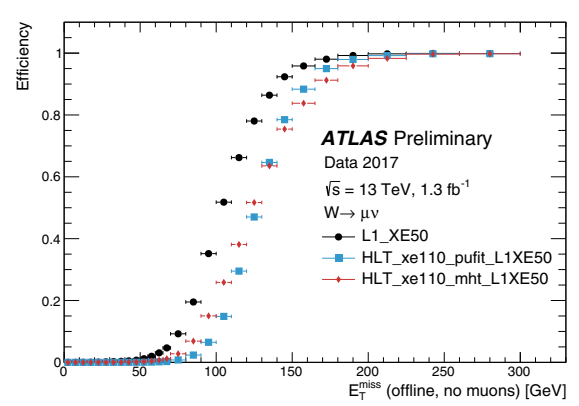

(a)

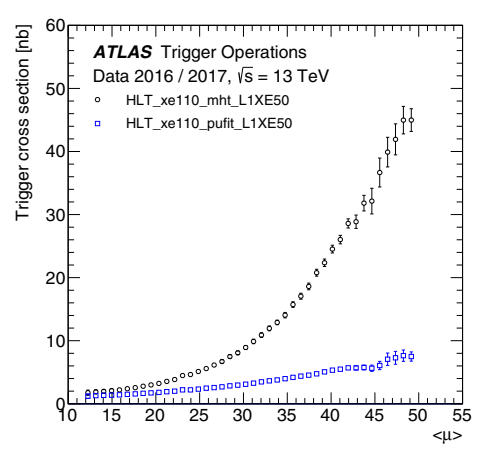

(b)

Figure 6. (a) Efficiency of L1, mht and pufit $E_{\mathrm{T}}^{\mathrm{miss}}$ triggers as a function of the offline $E_{\mathrm{T}}^{\text {miss }}[12]$. (b) Total trigger cross-section as a function of $\langle\mu\rangle$ for $m h t$ and pufit $E_{\mathrm{T}}^{\text {miss }}$ triggers with a $110 \mathrm{GeV}$ threshold [12].

\section{Conclusions}

Efficient triggering on hadronic signatures (jets and hadronic $E_{\mathrm{T}}^{\mathrm{miss}}$ ) is crucial for the ATLAS physics programme. The increasing luminosity during Run 2 of the LHC made this task particularly challenging. For the data taking periods of 2017 and 2018, the ATLAS jet and $E_{\mathrm{T}}^{\text {miss }}$ triggers implemented new strategies to face this challenge. These included improving jet calibration, exploiting the substructure of large- $R$ jets and using a much more pile-up insensitive $E_{\mathrm{T}}^{\mathrm{miss}}$ reconstruction algorithm.

\section{Acknowledgements}

E. G. is funded by Fundação para a Ciência e Tecnologia, through grant PD/BD/128231/2016.

\section{References}

[1] ATLAS Collaboration, JINST 3, S08003 (2008)

[2] ATLAS Collaboration,

https://twiki.cern.ch/twiki/bin/view/AtlasPublic/LuminosityPublicResultsRun2

[3] ATLAS Collaboration, Eur. Phys. J. C 76, no.10, 526 (2016)

[4] ATLAS Collaboration, Eur. Phys. J. C 77, no.5, 317 (2017)

[5] ATLAS Collaboration, Phys. Rev. D 96, no.7, 072002 (2017)

[6] ATLAS Collaboration, Eur. Phys. J. C 79, no.2, 135 (2019)

[7] D. Krohn, J. Thaler and L. T. Wang, JHEP 1002, 084 (2010)

[8] ATLAS Collaboration, ATL-DAQ-PUB-2018-001

[9] ATLAS Collaboration, ATL-DAQ-PUB-2018-002

[10] E. Simioni, arXiv:1406.4316 [physics.ins-det]

[11] ATLAS Collaboration, https://twiki.cern.ch/twiki/bin/view/AtlasPublic/JetTriggerPublicResults

[12] ATLAS Collaboration, https://twiki.cern.ch/twiki/bin/view/AtlasPublic/MissingEtTriggerPublicResults 